\title{
Anomalous chirality fluctuations in the initial stage of heavy ion collisions and parity odd bubbles
}

\author{
D. Kharzeev ${ }^{a}$, A. Krasnitz $^{b}$ and R. Venugopalan ${ }^{a, c}$ \\ ${ }^{a}$ Physics Department, Brookhaven National Laboratory, Upton, NY 11973, USA \\ E-mail: kharzeev@bnl.gov \\ ${ }^{b}$ CENTRA and Faculdade de Ciências e Tecnologia, \\ Universidade do Algarve, Campus de Gambelas, P-8000, Faro, Portugal \\ E-mail: krasnitz@ualg.pt \\ ${ }^{c}$ RIKEN-BNL Research Center, Brookhaven National Laboratory, Upton, NY 11973, \\ USA E-mail: raju@bnl.gov
}

\begin{abstract}
We compute numerically the topological charge distribution in the initial stage of a high energy heavy ion collision. This charge distribution is generated by Chern-Simons number fluctuations associated with the dynamics of strong classical fields in the initial state. The distribution is found to be quite narrow at RHIC and LHC energies reflecting a small value of the topological susceptibility. Thus the effective potential of classical fields is shallow in the $\theta$-direction likely creating favorable conditions for the subsequent generation of $\mathrm{P}$-odd bubbles.
\end{abstract}

PACS: 11.15.Ha, 12.38.Mh, 05.20.Gg, 05.40.+j.

Keywords: anomaly; lattice simulations; sphalerons; 


\section{Introduction}

In high energy heavy ion collisions, QCD matter is produced at very high energy densities. An issue that has aroused considerable interest is the possibility [1, 2] of creating metastable states that break the discrete global symmetries of parity $(P)$ and chargeparity $(C P)$ satisfied by the QCD Lagrangian $]$. There is a theorem by Vafa and Witten which states that there can be no stable parity violating phase of QCD [3]. The conditions of applicability of this theorem have attracted much attention recently [4, 5, 6]. The Vafa-Witten theorem does not however preclude the possibility of forming metastable domains that violate parity (and charge-parity) [1]. Furthermore, it has been suggested recently that the Vafa-Witten theorem may not be applicable at finite temperature [0].

Several experimental signatures have recently been suggested that would be sensitive to the formation of these $P$ and $C P$ odd metastable states [2]. Experimental searches are currently underway at RHIC. A preliminary report on their status was discussed at Quark Matter 2001 [8].

In the discussions of Refs. [1, 2], the formation of $P$ and $C P$-odd domains is studied in the context of the restoration of the axial $U(1)$ symmetry at finite temperature. A non-linear $\sigma$-model which incorporates the breaking of the $U(1)$ axial symmetry ?, the Di Vecchia-Veneziano-Witten Lagrangian [10], (VVW) is constructed and it is shown that there exist metastable states (corresponding to local minima of the potential) which spontaneously break $P$ and $C P$ [1]. The likelihood of forming long-lived metastable states depends on the coefficient $a \sim m_{\eta^{\prime}}^{2}$ of the anomaly term in the VVW Lagrangian - they are more likely when $a(T) \rightarrow 0$ as the temperature approaches the deconfinement temperature, $T \rightarrow T_{c}$. There is evidence from lattice simulations that this is the case. The anomaly term is proportional to the topological susceptibility and lattice data suggest that the topological susceptibility for both quenched and full QCD drops rapidly in the vicinity of $T_{c}$ and is consistent with zero by $T>1.5 T_{c}\left(1.2 T_{c}\right)$ for $N_{f}=2\left(N_{f}=4\right)$ [1]. Further, large $N_{c}$ analyses suggest that $a(T) \rightarrow 0$ as $T \rightarrow T_{c}$ [1, 2, 13, 12].

In Ref. [2], the decay of $P$ and $C P$ odd metastable states into pions is described by the Wess-Zumino-Witten term [14] in the chiral Lagrangian. This term preserves the net chirality or handedness generated in the $P$ and $C P$ odd domains. The magnitude of the net asymmetry produced by this mechanism in heavy ion collisions is estimated to be $\sim 10^{-3}[2]$. The signatures of $\mathrm{P}$ and $\mathrm{CP}$ odd domains were investigated also in Refs. [15, 16, 17], and include an enhancement in the $\eta$ and $\eta^{\prime}$ yields, generically associated with the $U_{A}(1)$ restoration 18 .

It is of great interest to investigate how the topological susceptibility is affected by the dynamical conditions generated in the early stage of a heavy ion collision. Immediately after a high energy nuclear collision, as a consequence of the phenomenon of gluon saturation [19], large color electric and magnetic fields are generated. These can in principle produce a large amount of topological charge through the mechanism of sphaleron

\footnotetext{
${ }^{1}$ This is true in the absence of a $\theta$-term which would break $P$ and $C P$ explicitly. We will assume that $\theta=0$ here.

${ }^{2}$ For a recent study of $U_{A}(1)$ restoration in the linear sigma model, see Ref. [9].
} 
transitions already in the initial stage of the heavy ion collision. However, as we will argue below, the boost invariance of the initial stage of the heavy ion collision suppresses sphaleron transitions. The primary mechanism for the generation of topological charge at the early stage is by fluctuations of the color electric and magnetic fields. For a translationally invariant system, the fluctuations in the topological charge determine the topological susceptibility.

In this paper, we estimate the size of topological charge fluctuations in the early stage of a heavy ion collision. Our computation is performed within the framework of a classical effective field theory (EFT) [20, 21] approach to small $x$ physics. In this approach, parton distributions in a nucleus before the collision form a color glass condensate (CGC) [22, 23]. The shattering of the color glass condensate and subsequent multiparticle production in high energy scattering has been discussed for eA [20], pA [29] and for nuclear collisions [24, 30]. For nuclear collisions, the problem can be formulated in the EFT [24] and the temporal evolution of gluons produced after the collisions can be solved for numerically [26]. The initial energy [27] and number [28] of produced gluons have been computed previously in this approach. The CGC idea has been shown to be consistent with many features of data from heavy ion collisions [30, 31].

We find in the CGC model that the fluctuations in topological charge are quite small at RHIC and LHC energies. The root mean squared topological charge is only one unit per two units of rapidity at RHIC energies and one unit per unit of rapidity at LHC energies. Our results suggest that the form of the effective potential in the $\theta$-direction is quite shallow at early times. This result makes favorable the formation of $P$ and $C P$ odd bubbles at later times - namely, the scenario that was first discussed in Refs. [1, 2].

Recently instanton/sphaleron approaches to multiparticle production have been investigated [32, 33, 34, 35]. In Ref. [34] it was shown that in the initial stage of nuclear collisions instanton transitions (and therefore the topological susceptibility) are severely suppressed by strong classical gluon fields. Shuryak has suggested that the dominant mechanism for particle production in nuclear collisions may be through the decay of sphalerons [35]. If so, a likely consequence is that the ensuing sphaleron transitions will generate large $P$ and $C P$ violation already in the initial stage of the nuclear collision. In our approach, however, sphaleron transitions are suppressed by strict boost invariance. Sphaleron transitions, if they occur, will take place at a later stage when the strict boost invariance of the initial state is lost. We have estimated the topological charge generated by sphaleron transitions in a hot gluon plasma and find, for RHIC energies, that it is large compared to the topological charge generated in the initial stage by classical fields.

The paper is organized as follows. In section 2, we briefly review the CGC picture of classical gluon production in heavy ion collisions. The no-go theorem for sphaleron transitions for a strictly boost invariant system is derived in section 3. Numerical results for the Chern-Simons charge are presented in section 4. Its relation to empirical observables is also discussed there. In section 5, we discuss estimates for $P$ and $C P$ violation from sphaleron transitions at finite temperature in equilibrium hot gluodynamics. We also comment on the implications of these results (versus those in the boost invariant CGC picture) for the heavy ion experiments. 


\section{Classical gluon production in nuclear collisions}

In a high energy heavy ion collision, the dynamics of the central rapidity region is determined by the small $x$ modes in the individual nuclei before the collision. At small $x$, the gluon density grows rapidly - however, repulsive effects soon become important and slow down the growth of the gluon density. This phenomenon is called saturation and has been discussed extensively in the literature [19]. The occupation number of gluons in the saturated regime is proportional to $1 / \alpha_{s}\left(\Lambda_{s}\right)$ evaluated at a saturation scale $\Lambda_{s}$. If $\Lambda_{s} \gg \Lambda_{Q C D}$, the occupation number is large and classical methods may be applied to study parton distributions in the nuclei [20, 21]. It has been shown recently that a RG-improved generalization of this classical effective field theory (EFT) reproduces several key results in small- $x$ QCD [21, 23]. The classical EFT was first applied to the study of collisions of large nuclei by Kovner, McLerran and Weigert [24]. The model, as applied to nuclear collisions, may be summarized as follows. The colliding nuclei are idealize to travel along the light cone The high- $x$ and the low- $x$ modes in the nuclei are treated separately. Th former corresponds to valence quarks and hard sea partons and are considered recoilless sources of color charge For each of the large Lorentz-contracted nuclei (for simplicity, we will consider only collisions of identical nuclei), this results in a static Gaussian distribution of their color charge density $\rho_{1,2}$ in th transverse plane

$$
P([\rho]) \propto \exp \left[-\frac{1}{2 \Lambda_{s}^{2}} \int \mathrm{d}^{2} r_{t} \rho_{1,2}^{2}\left(r_{t}\right)\right] .
$$

The variance $\Lambda_{s}$ of the color charge distribution is the only dimensionful parameter of the model, apart from the linear size $L$ of the nucleus. For central impact parameters, $\Lambda_{s}$ can be estimated in terms of single-nucleon structure functions [25]. It is assumed, in addition, that the nucleus is infinitely thin in the longitudinal direction. Under this simplifying assumption, the resulting gauge fields are explicitly boost-invariant.

The small $x$ fields are then described by the classical Yang-Mills equations

$$
D_{\mu} F_{\mu \nu}=J_{\nu}
$$

with the random sources on the two light cones: $J_{\nu}=\sum_{1,2} \delta_{\nu, \pm} \delta\left(x_{\mp}\right) \rho_{1,2}\left(r_{t}\right)$. The two signs correspond to two possible directions of motion along the beam axis $z$. As shown by Kovner, McLerran and Weigert (KMW) [24, low- $x$ fields in the central region of the collision obey sourceless Yang-Mills equations (this region is in the forward light cone of both nuclei) with the initial conditions in the $A_{\tau}=0$ gauge given by

$$
A^{i}=A_{1}^{i}+A_{2}^{i} ; \quad A^{ \pm}= \pm \frac{i g}{2} x^{ \pm}\left[A_{1}^{i}, A_{2}^{i}\right] .
$$

Here the pure gauge fields $A_{1,2}^{i}$ are solutions of (11) for each of the two nuclei in the absence of the other nucleus.

In order to obtain the resulting gluon field configuration at late proper times, one needs to solve (四) with the initial condition (2). Since the latter depends on the random 
color source, averages over realizations of the source must be performed. KMW showed that in perturbation theory the gluon number distribution by transverse momentum (per unit rapidity) suffers from an infrared divergence and argued that the distribution must have the form

$$
n_{k_{\perp}} \propto \frac{1}{\alpha_{s}}\left(\frac{\Lambda_{s}}{k_{\perp}}\right)^{4} \ln \left(\frac{k_{\perp}}{\Lambda_{s}}\right)
$$

for $k_{\perp} \gg \Lambda_{s}$. The log term clearly indicates that the perturbative description breaks down for $k_{\perp} \sim \Lambda_{s}$.

A reliable way to go beyond perturbation theory is to re-formulate the EFT on a lattice by discretizing the transverse plane. The resulting lattice theory can then be solved numerically. We shall not dwell here on the details of the lattice formulation, which is described in detail in Ref. [26, 27]. Keeping in mind that $\Lambda_{s}$ and the linear size $L$ of the nucleus are the only physically interesting dimensional parameters of the model [22], we can write any dimensional quantity $q$ as $\Lambda_{s}^{d} f_{q}\left(\Lambda_{s} L\right)$, where $d$ is the dimension of $q$. All the non-trivial physical information is contained in the dimensionless function $f_{q}\left(\Lambda_{s} L\right)$. We can estimate the values of the product $\Lambda_{s} L$ which correspond to key collider experiments. Assuming Au-Au collisions, we take $L=11.6 \mathrm{fm}$ (for a square nucleus!) and estimate the saturation scale $\Lambda_{s}$ to be $2 \mathrm{GeV}$ for RHIC and $4 \mathrm{GeV}$ for LHC [25]. Also, we have approximately $g=2$ for energies of interest. The rough estimate is then $\Lambda_{s} L \approx 120-150$ for RHIC and $\Lambda_{s} L \approx 240-300$ for LHC. Since the gluon distribution in nuclei is not known to great precision, there is a considerable systematic uncertainty in these estimates.

This model has been applied recently to study classical gluon production. The energy and number distributions have been computed numerically, and the dependence of these quantities on $\Lambda_{s}$ has been determined [27, 28]. We will now apply the model to compute the Chern Simons number generated in the early stages of a heavy ion collision.

\section{Boost invariance and Chern Simons number}

In general, the Chern-Simons (CS) number changes under a gauge transformation by an integer equal to the winding number of that transformation. Since the potential of the theory is invariant under all gauge transformations, there exists a direction in the functional space along which the potential is periodic. The system can move along that direction arbitrarily far from its initial configuration. Such motion generates arbitrarily large changes of the CS number or, equivalently, arbitrarily large values of topological charge. Dynamical, real time, processes corresponding to nontrivial gauge transformations are called sphaleron transitions. In a large space-time volume these transitions proceed independently in causally unrelated regions. If, in addition, the space-time is translation-invariant, the topological charge for a large space-time volume is a random walk:

$$
\left\langle\left(N_{\mathrm{cs}}(t)-N_{\mathrm{cs}}\left(t^{\prime}\right)\right)^{2}\right\rangle=\Gamma V\left(t-t^{\prime}\right)
$$


where $\Gamma$ is known as the sphaleron transition rate, or as the topological susceptibility. Here, and in the following, $\langle\ldots\rangle$ means an ensemble average: the thermal ensemble in thermal equilibrium, and the ensemble of (central) nuclear collision events in the case of interest here. This random walk behavior was indeed observed in numerical simulations of $\mathrm{SU}(2)$ [36] and $\mathrm{SU}(3)$ [37] Yang-Mills theories at high temperature.

The dynamics of the CS number in the central region of a nuclear collision differs from the generic case in two ways. Firstly, the time translation invariance is broken by the instance of the collision itself. Secondly, and more importantly, there are no nontrivial boost-invariant gauge transformations. Consequently, for boost-invariant configurations, such as those in the central region, the potential is not periodic, and there can be no sphaleron transitions. Instead, one expects the CS number to fluctuate in the vicinity of zero.

In order to make this second point explicit, we shall now examine the form of the CS number functional for boost-invariant configurations, and show it to be invariant under all boost-invariant gauge transformations. It will then follow that, in this special case, the CS number cannot be changed by a dynamical process equivalent to a gauge transformation, i.e., that sphaleron transitions are prohibited for a boost invariant system.

It is convenient for us to use the coordinates $\left(\vec{r}_{t}, \tau, \eta\right)$. Here $\tau=\sqrt{2 x^{+} x^{-}}$is the proper time, $\eta=\ln \left(x^{+} / x^{-}\right) / 2$ is the space-time rapidity, and $\vec{r}_{t} \equiv(x, y)$ is the position in the transverse plane. For the components of the gauge potential, the boost invariance assumption (see the discussion immediately prior to Eq. 1) means that

$$
\vec{A}_{t}\left(\tau, x_{t}, \eta\right)=\overrightarrow{A_{t}}\left(\tau, x_{t}\right) ; A_{\eta}\left(\tau, x_{t}, \eta\right) \equiv \Phi\left(\tau, x_{t}\right)
$$

and the system effectively becomes $(2+1)$-dimensional, with $A_{\eta}$ becoming an adjoint scalar field. It is also convenient to remove the fourth component of $A$ by imposing the gauge condition $A^{\tau}=0$.

Now consider the quantity

$$
\nu=\frac{1}{16 \pi^{2}} \int \mathrm{d}^{2} x_{t} \Phi^{a} B^{a}
$$

where

$$
B^{a}=F_{x y}^{a}=\partial_{x} A_{y}^{a}-\partial_{y} A_{x}^{a}+g \epsilon^{a b c} A_{x}^{b} A_{y}^{c}
$$

is the component of the magnetic field perpendicular to the transverse plane. This quantity is obviously invariant under all rapidity independent gauge transformations. In order to verify that $\nu$ is the CS number per unit rapidity, we will compute its proper time derivative (denoted by a dot in the following) and show it to be equal to the topological charge density integrated over the transverse plane. We recall that the topological charge density is given by

$$
\frac{1}{16 \pi^{2}} \vec{E} \cdot \vec{B}
$$


$\vec{E}^{a}$ and $\vec{B}^{a}$ being the color electric and magnetic fields, respectively; the anomaly equation relates this quantity to the divergence of the chiral current.

To this end, it suffices to write out $\dot{\nu}$ explicitly, using (7), and integrate the terms containing $\partial_{x} \dot{A}_{y}^{a}$ and $\partial_{y} \dot{A}_{x}^{a}$ by parts in the transverse plane, assuming that the corresponding boundary terms vanish. The result is

$$
\dot{\nu}=\frac{1}{16 \pi^{2}} \int \mathrm{d}^{2} x_{t}\left(\dot{\Phi}^{a} B^{a}+\dot{A}_{x}^{a} D_{y}^{a} \Phi-\dot{A}_{y}^{a} D_{x}^{a} \Phi\right),
$$

where $D_{i}^{a} \Phi$ is the covariant derivative: $D_{i}^{a} \Phi \equiv \partial_{i} \Phi^{a}+\epsilon^{a b c} A_{i}^{b} \Phi^{c}$. To complete the proof, we note that (a) for boost invariant configurations, the integrand of (90) is equal to $\tau \vec{E}^{a} \cdot \vec{B}^{a}$; and $(b)$ the space-time volume element in the $\tau, \eta, \vec{r}_{t}$ coordinates is $\mathrm{d}^{4} x=\tau \mathrm{d} \eta \mathrm{d} \tau \mathrm{d}^{2} x_{t}$. Hence we have shown that the proper time variation of $\nu$ in a boost invariant process gives the topological charge per unit rapidity of the process. If any such process is equivalent to a gauge transformation, it leaves $\nu$ unchanged, the corresponding topological charge vanishes, and no sphaleron transitions are possible.

In the EFT description of nuclear collisions the scalar field $\Phi$ vanishes at $\tau=0$ [26], and, therefore, $\nu(\tau=0)=0$. In other words, the topological charge of the process at any given $\tau$ is simply $\eta \nu(\tau)$, where $\eta$ is the rapidity extent of the process. Since the EFT has a parity-even action, both signs of $\nu(\tau)$ are equally probable, and the ensemble average of $\nu(\tau)$ vanishes. Our principal object of interest is then $\left\langle\nu^{2}(\tau)\right\rangle$. In the absence of sphaleron transitions, we cannot expect the proper time evolution of $\nu$ to resemble a random walk. Indeed, as we shall see in the next section, $\left\langle\nu^{2}(\tau)\right\rangle$ approaches a constant at large $\tau$.

Strictly speaking, because of the strict boost invariance built into our approach, $\left\langle\nu^{2}(\tau)\right\rangle$ per unit space-time volume cannot be interpreted as the topological susceptibility. Nevertheless, the computed $\left\langle\nu^{2}(\tau)\right\rangle$ is a measure of the contribution of classical fields to the topological susceptibility. As will be discussed further in Section 5, the likelihood of forming long-lived P and CP odd domains depends sensitively on this quantity.

\section{Numerical results}

We use a lattice formulation of the EFT developed in [26, 22, 28]. As explained in these references, the key dimensionless parameter for those observables which exist in the continuum limit is $\Lambda_{s} L$, where $L$ is the radius of the nucleus (assuming a square nucleus, $\left.L^{2}=\pi R^{2}\right)$. The value of $\Lambda_{s}$, expressed in units of the lattice spacing $a$, indicates how close the system is to the continuum limit. In the current study, for an $\mathrm{SU}(2)$ gauge theory [ pe set $\Lambda_{s} a=0.29$. The CS number $\nu$ is a dimensionless quantity, whose

\footnotetext{
${ }^{3}$ We study an SU(2) gauge theory since it is simpler to simulate numerically. The physically more interesting case of an SU(3) gauge theory was studied recently and gluon number distributions computed [47]. No significant qualitative differences from the $\mathrm{SU}(2)$ case were found for the energies of interest.
} 


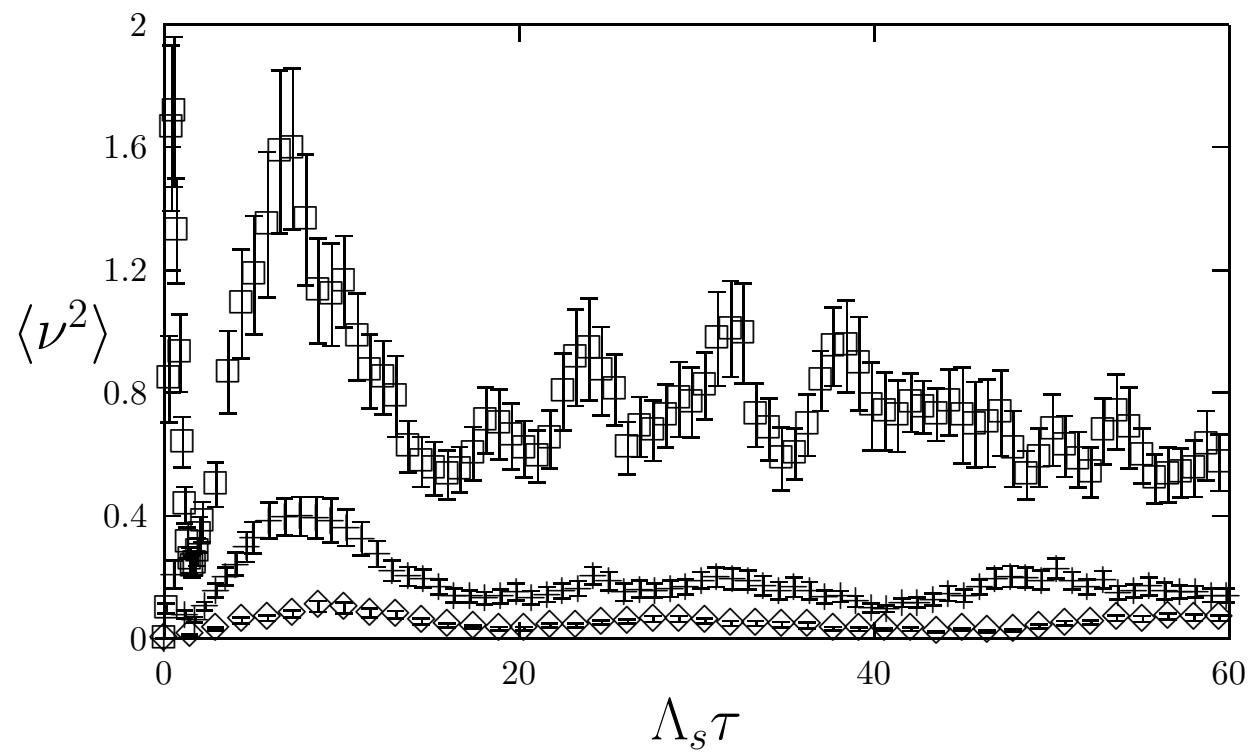

Figure 1: Average squared topological charge $v s$ the proper time (in units of $\Lambda_{s}^{-1}$ ) for $\Lambda_{s} L=74.2$ (diamonds), 148.4 (pluses), and 297 (squares). The latter two values of $\Lambda_{s} L$ correspond to the RHIC $\left(\Lambda_{s}=2 \mathrm{GeV}\right)$ and LHC $\left(\Lambda_{s}=4 \mathrm{GeV}\right)$ regimes respectively.

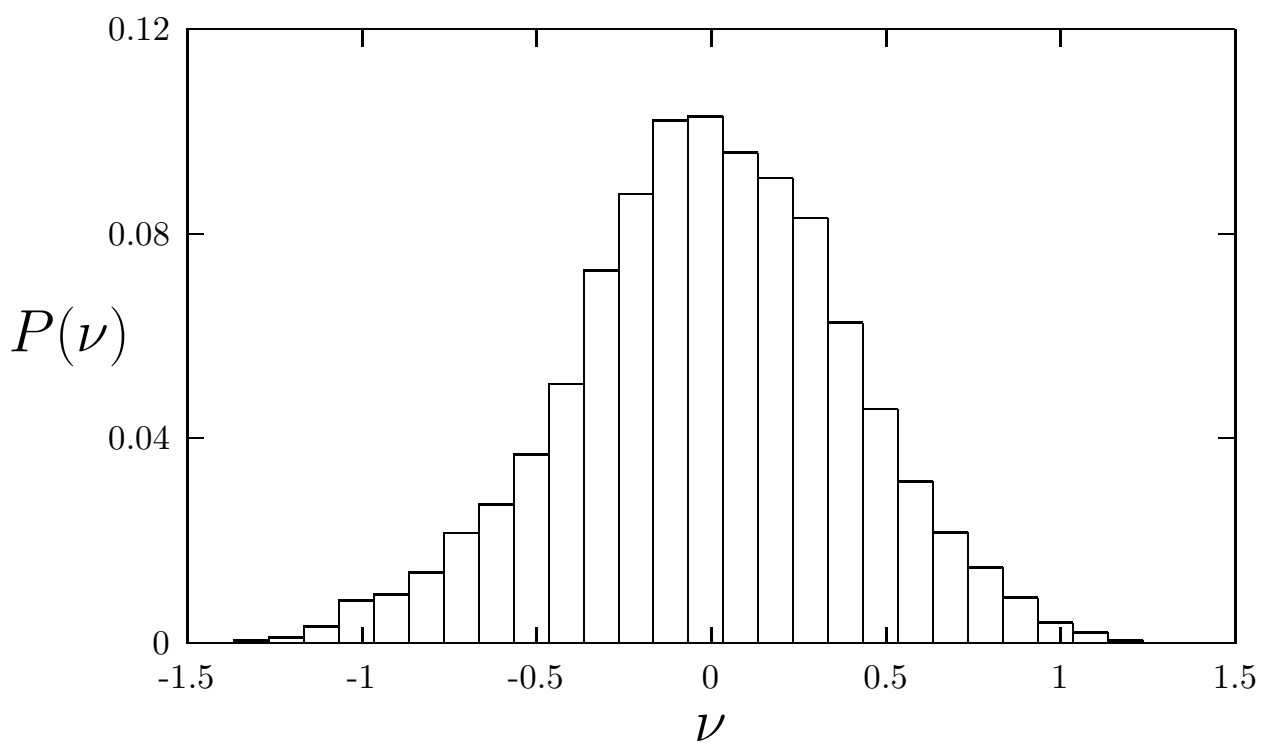

Figure 2: Probability distribution of $\nu$ in the proper time interval $30 \leq \Lambda_{s} \tau \leq 65$ for $\Lambda_{s} L=148.4$. These corresponds to $3 \mathrm{fm} \leq \tau \leq 6 \mathrm{fm}$ for an estimated value $\Lambda_{s}=2 \mathrm{GeV}$ for RHIC energies. 
ultraviolet properties are similar to those of the total gluon number $N$, studied in [28]. In that study, we determined that discretization artifacts are very small for $N$ at $\Lambda_{s} a=0.29$.

We examined the proper time evolution of $\nu$ for three values of $\Lambda_{s} L$, namely, 74.2, 148.4, and 297. The last two of these correspond roughly to central $\mathrm{Au}-\mathrm{Au}$ collisions in the RHIC and LHC regimes, respectively. In each of the three cases, we generated about 80 independent initial configurations and solved the classical equations of motion for the duration of proper time of at least $60 / \Lambda_{s}$ (this corresponds to $6 \mathrm{fm}$ for RHIC). The average proper time histories of $\nu^{2}$ in each case are presented in Figure 1. Evidently, $\nu$ varies over a much longer time scale, compared to other global quantities, such as the total energy and the total particle number [27, 28]. Nevertheless, $\left\langle\nu^{2}(\tau)\right\rangle$ does approach a constant value at late $\tau$. That constant value is a rapidly growing function of $\Lambda_{s} L$. Since the dynamics considered here is exactly boost-invariant, $\left(\left\langle\nu^{2}(\tau)\right\rangle\right)^{1 / 2}$ is a measure of the anomalous chirality violation per unit rapidity in the initial stage of a central collision.

To estimate the magnitude of the effect, we took the proper time average of $\left\langle\nu^{2}(\tau)\right\rangle$ between $\Lambda_{s} \tau=30$ and $\Lambda_{s} \tau=65$. The results are summarized in Table 1 . If we take the experimental range of two units of rapidity, it follows that for RHIC we have approximately one unit of the topological charge squared for two units of rapidity while at LHC one may have two units of topological charge squared in the same rapidity interval. Note that $\left\langle\nu^{2}\right\rangle$ is approximately proportional to $\left(\Lambda_{s} R\right)^{2}$, i.e., to the transverse area in units of the nonperturbative scale $\Lambda_{s}$. This means that dynamical processes contributing to the topological charge become uncorrelated at spatial separations exceeding $\Lambda_{s}$, and the resulting fluctuations of $\nu$ add up randomly.

\begin{tabular}{|lrrr|}
\hline$\Lambda_{s} R$ & 41.9 & 83.7 & 167.5 \\
$\left\langle\nu^{2}\right\rangle$ & $0.046 \pm 0.004$ & $0.16 \pm 0.01$ & $0.69 \pm 0.05$ \\
\hline
\end{tabular}

Table 1: The average value of $\left\langle\nu^{2}\right\rangle$, tabulated as a function of the dimensionless parameter $\Lambda_{s} R$.

As we have explained in the preceding sections, there is no CS number diffusion in a boost invariant setting, nor is there a ground state degeneracy. It is for this reason that $\left\langle\nu^{2}(\tau)\right\rangle$ approaches a constant at large $\tau$, instead of growing linearly, as would be the case if sphaleron transitions were permitted. Also, as Figure 2 illustrates, there is no special significance to integer values of $\nu$ other than zero, which in a general case correspond to degenerate minima of the potential. 


\section{Comparison with other estimates of the topologi- cal susceptibility and experimental ramifications}

Thus far, we have assumed that strict boost invariance holds in heavy ion collisions at very high energies. If strict boost invariance does not hold, sphaleron transitions are allowed. Whether sphaleron transitions turn on slowly or rapidly when strict boost invariance is relaxed is not clear. A way to answer this question is to introduce a small perturbation $a\left(x_{t}, \eta, \tau\right)$, namely,

$$
\tilde{A}\left(x_{t}, \eta, \tau\right)=A\left(x_{t}, \tau\right)+a\left(x_{t}, \eta, \tau\right),
$$

in the Yang-Mills equations and to study how it evolves. While the Yang-Mills equations equations can be linearized with respect to $a\left(x_{t}, \eta, \tau\right)$, such a study has to be performed numerically, since $A\left(x_{t}, \tau\right)$ is not known analytically 48 .

Let us assume that the system does thermalize and strict boost invariance of field configurations is lost . We then need an estimate of the sphaleron transition rate in a hot gluon plasma. To simplify the setup, we shall first ignore dynamical fermions and assume that the temperature $T$ is much larger than any other dimensional scale in the problem. It then follows from dimensional considerations 38 that the rate is proportional to $T^{4}$ However, the dependence of the rate on the coupling constant $g$ is less obvious. In the weak-coupling regime the sphaleron transitions are dominated by soft modes, with momenta of the order of $g^{2} T$. The time scale for the transition depends on how important is the interaction between these degrees of freedom and hard thermal modes. A series of perturbative studies [44, 45], performed at extremely weak coupling $(\ln (1 / g) \gg 1)$, suggest that this interaction sets the time scale for sphaleron transitions to $\left(g^{4} T\right)^{-1}$ up to logarithmic corrections. Then the coupling constant dependence is $\Gamma \propto \alpha_{s}^{5} T^{4}$, and a recent numerical estimate gives [37]

$$
\Gamma_{s p h}=108 \alpha_{s}^{5} T^{4}
$$

On the other hand, there are indications that, away from the asymptotically weak coupling regime, the relevant time scale for the soft modes is $\left(g^{2} T\right)^{-1}$ 46. Should this be the case, the numerical estimate [37] should be re-interpreted to give

$$
\Gamma_{s p h} \approx 8 \alpha_{s}^{4} T^{4}
$$

In the following, we will use both estimates.

For a hot plasma (without dynamical fermions) the potential is periodic and one generates topological charge via a random walk. One has

$$
\left\langle\Delta Q_{5}^{2}\right\rangle=\int_{0}^{\tau} d^{4} x \Gamma_{s p h}
$$

\footnotetext{
${ }^{4}$ Boost invariance may still be preserved when one averages over events.
} 
where $d^{4} x=d^{2} x_{t} \tau d \tau d \eta$. In an expanding boost invariant plasma, the temperature decreases with time. For an isentropic expansion [39] one has $T \propto \tau^{-1 / 3}$. More precisely,

$$
T=\left[\frac{90}{4 \pi^{2}} \frac{d S}{d \eta} \frac{1}{L^{2} g_{d}} \frac{1}{\tau}\right]^{1 / 3},
$$

where $g_{d}$ is the degeneracy factor and $d S / d \eta$ is the entropy per unit rapidity. From Eq. 13 and Eq. 14, we obtain

$$
\frac{d\left\langle\Delta Q_{5}^{2}\right\rangle}{d \eta}=1.5 A \alpha_{s}^{n}\left(\frac{\tau_{f}}{L}\right)^{2 / 3}\left[\frac{d S}{d \eta} \frac{1}{g_{d}} \frac{90}{4 \pi^{2}}\right]^{4 / 3},
$$

where $A$ and $n$ are correspond to the different coefficient and power respectively in the two sphaleron transition rates. At RHIC energies, $d S / d \eta \sim 3.6 \times 1000, \alpha_{s} \sim 0.3$, $\tau_{f} \approx L \sim 10 \mathrm{fm}, g_{d}=16$. Then from the sphaleron transition rate in Eq. 11, and from Eq. 15, we find for an expanding plasma,

$$
\left.\frac{d\left\langle\Delta Q_{5}^{2}\right\rangle^{(1)}}{d \eta}\right|_{R H I C} \approx 1600 ;\left.\frac{d\left\langle\Delta Q_{5}\right\rangle_{R M S}^{(1)}}{d \eta}\right|_{R H I C} \approx 40 .
$$

Here RMS denotes root mean square. Similarly, for the sphaleron transition rate in Eq. 12, and Eq. 15, we have

$$
\left.\frac{d\left\langle\Delta Q_{5}^{2}\right\rangle^{(2)}}{d \eta}\right|_{R H I C} \approx 400 ;\left.\frac{d\left\langle\Delta Q_{5}\right\rangle_{R M S}^{(2)}}{d \eta}\right|_{R H I C} \approx 20 .
$$

Though these estimates of the topological charge generated by sphaleron transitions differ from each other they both are significantly larger than the topological charge squared obtained from boost invariant classical fields at RHIC (and LHC). In this paper, we have considered the case of pure gluodynamics; the effects due to dynamical fermions had been addressed in Refs. [40, 41, 43].

We now come to the phenomenological implications of our results. As discussed previously, the likelihood of long-lived $\mathrm{P}$ and $\mathrm{CP}$ violating metastable states in heavy ion collisions [1, 2] depends sensitively on the coefficient $a$ of the anomaly term in the VVW Lagrangian. This coefficient is proportional to the topological susceptibility. The likelihood of metastable states is greater for smaller values of $a$. Lattice and large $N_{c}$ calculations suggest that the topological susceptibility is indeed small for $T>T_{c}$ The previous estimates did not include the possibility of contributions from fluctuations in classical fields or from sphaleron transitions. A difficult problem is how to convert the estimates for generating non-zero topological charge into one for $P$ and $C P$ odd asymmetries for measured pions 9 . We will not address this problem directly here. Instead, since the quantity $\left\langle\nu^{2}\right\rangle$ that we compute here is closely related to the topological susceptibility, we can relate our estimate to those of Refs. [1, 2]. Our estimates here

\footnotetext{
${ }^{5}$ We speculate that one may construct, akin to $\vec{L} \cdot \vec{S}$ coupling, invariants resulting from the coupling of the vorticity of CS number to that of the vorticity of collective flow in an expanding plasma.
} 
suggest that the contribution to $a$ from classical fields is small and would not therefore affect the estimate of Ref. [2]. On the other hand, the contribution from sphaleron transitions might be potentially large. Thus if the RHIC experiments see a large $P$ and $C P$ violating effect, it may also be due to sphalerons 35 .

Our results depend on the strong boost invariance assumption, namely, each event is boost invariant. At central rapidities, the measured spectra look boost invariant 49 on average. However, what is relevant to our assumption is the requirement that the spectra are boost invariant on an event by event basis. One way to ensure this is to compute the standard deviation from strict boost invariance in one unit of rapidity about mid-rapidity and check if it is small. Such studies are underway but no conclusive results have been published yet [50]. As one goes to larger rapidities, the boost invariance assumption breaks down. The no-go theorem forbidding sphaleron transitions is violated and one may therefore see larger effects at non-central rapidities (in the $P$ and $C P$ odd observables currently being investigated) due to sphaleron transitions. It will be very interesting to see if this prediction can be tested in the on-going experiments at RHIC.

\section{Acknowledgments}

We thank Dietrich Bödeker, Greg Carter, Yuri Kovchegov, Eugene Levin, Larry McLerran, Emil Mottola, Rob Pisarski, Mikhail Shaposhnikov and Edward Shuryak for useful discussions. D.K. and R.V. were supported by DOE Contract No. DE-AC0298CH10886. A.K. and R.V acknowledge the support of Portuguese FCT, under grants CERN/P/FIS/15196/1999 and CERN/P/FIS/40108/2000. Finally, R.V. would like to acknowledge the support of RIKEN-BNL, and A.K. would like to thank the Nuclear Theory Group, BNL, for its hospitality.

\section{References}

[1] D. Kharzeev, R. D. Pisarski and M. H. Tytgat, Phys. Rev. Lett. 81, 512 (1998).

[2] D. Kharzeev and R. D. Pisarski, Phys. Rev. D 61, 111901 (2000).

[3] C. Vafa and E. Witten, Phys. Rev. Lett. 53, 535 (1984); Nucl. Phys. B 234, 173 (1984).

[4] V. Azcoiti and A. Galante, Phys. Rev. Lett. 83, 1518 (1999).

[5] S. Sharpe and R. Singleton Jr., Phys. Rev. D 58074510 (1998).

[6] X. Ji, hep-ph/0108162.

[7] T. Cohen, Phys. Rev. D 64047704 (2001). 
[8] A. Chikanian, L.E. Finch, R.S. Longacre, J. Sandweiss, J.H. Thomas, presented at Quark Matter 2001, Stony Brook, NY, January 16th-21st, (2001), and work in progress.

[9] J. T. Lenaghan, Phys. Rev. D 63, 037901 (2001).

[10] P. Di Vecchia and G. Veneziano, Nucl. Phys. B 171, 253 (1980); E. Witten, Annals Phys. 128, 363 (1980).

[11] B. Alles, M. D’Elia and A. Di Giacomo, Phys. Lett. B 483, 139 (2000).

[12] I. Affleck, Nucl. Phys. B 162, 461 (1980).

[13] R. D. Pisarski and M. Tytgat, hep-ph/9702340.

[14] J. Wess and B. Zumino, Phys. Lett. B37, 95 (1971); E. Witten, Nucl. Phys. B223, $422(1983)$.

[15] R. Buckley, T. Fugleberg, A. Zhitnitsky, Phys. Rev. Lett. 84, 4814 (2000); Phys. Rev. C63, 034602 (2001).

[16] D. Ahrensmeier, R. Baier, M. Dirks, Phys. Lett. B484, 58 (2000).

[17] A.K. Chaudhuri, hep-ph/0012047; hep-ph/0109211.

[18] J. Kapusta, D. Kharzeev, L. McLerran, Phys. Rev. D53, 5028 (1996); Z. Huang and X.-N. Wang, Phys. Rev. D53, 5034 (1996).

[19] L. V. Gribov, E. M. Levin and M. G. Ryskin, Phys. Rept. 100, 1 (1983); A. H. Mueller and J. Qiu, Nucl. Phys. B 268, 427 (1986); J.P. Blaizot and A.H. Mueller, Nucl. Phys. B289, 847 (1987); A. H. Mueller, hep-ph/9911289.

[20] L. McLerran and R. Venugopalan, Phys. Rev. D 49, 2233 (1994); 49, 3352 (1994); D 50, 2225 (1994); D 59, 094002 (1999); Yu. V. Kovchegov and L. McLerran, Phys. Rev. D 60, 054025 (1999).

[21] J. Jalilian-Marian, A. Kovner, L. McLerran and H. Weigert, Phys. Rev. D 55, 5414 (1997); Yu. V. Kovchegov, Phys. Rev. D 54, 5463 (1996); J. Jalilian-Marian, A. Kovner, A. Leonidov and H. Weigert, Nucl. Phys. B 504, 415 (1997); J. JalilianMarian, A. Kovner and H. Weigert, Phys. Rev. D 59, 014015 (1999).

[22] R. V. Gavai and R. Venugopalan, Phys. Rev. D 54, 5795 (1996).

[23] E. Iancu, A. Leonidov and L. McLerran, hep-ph/0011241; hep-ph/0102009; hep$\mathrm{ph} / 0103032$.

[24] A. Kovner, L. McLerran and H. Weigert, Phys. Rev. D 52, 3809 (1995); D 52, 6231 (1995); Yu. V. Kovchegov and D. H. Rischke, Phys. Rev. C 56, 1084 (1997).

[25] M. Gyulassy and L. McLerran, Phys. Rev. C 56, 2219 (1997). 
[26] A. Krasnitz and R. Venugopalan, Nucl. Phys. B 557, 237 (1999).

[27] A. Krasnitz and R. Venugopalan, Phys. Rev. Lett. 84, 4309 (2000).

[28] A. Krasnitz and R. Venugopalan, Phys. Rev. Lett. 86, 1717 (2001).

[29] Yu. V. Kovchegov and A. H. Mueller, Nucl. Phys. B 529, 451 (1998); A. Dumitru and L. McLerran, hep-ph/0105268.

[30] D. Kharzeev and M. Nardi, Phys. Lett. B 507, 121 (2001); D. Kharzeev and E. Levin nucl-th/0108006.

[31] L. McLerran and J. Schaffner-Bielich, hep-ph/0101133; J. Schaffner-Bielich, D. Kharzeev, L. D. McLerran and R. Venugopalan, nucl-th/0108048

[32] D. Kharzeev and E. Levin, Nucl. Phys. B578, 351 (2000); D. E. Kharzeev, Yu. V. Kovchegov and E. M. Levin Nucl. Phys. A 690, 621 (2001).

[33] E. V. Shuryak Phys. Lett. B 486, 378 (2000); E. V. Shuryak and I. Zahed Phys. Rev. D 62, 085014 (2000); M. A. Nowak, E. V. Shuryak and I. Zahed Phys. Rev. D 64, 034008 (2001)

[34] D. Kharzeev, Yu. Kovchegov, and E. Levin, hep-ph/0106248.

[35] E. V. Shuryak, hep-ph/0101269.

[36] J. Ambjorn and A. Krasnitz, Phys. Lett. B 362, 97 (1995); Nucl. Phys. B 506, 387 (1997).

[37] G. D. Moore, Phys. Lett. B 412, 359 (1997).

[38] P. Arnold and L. McLerran, Phys. Rev. D 36, 581 (1987); Phys. Rev. D 37, 1020 (1988).

[39] J. D. Bjorken, Phys. Rev. D 27, 140 (1983); G. Baym, Phys. Lett. B 138, 18 (1984).

[40] L. McLerran, E. Mottola and M. Shaposhnikov, Phys. Rev. D 43, 2027 (1991).

[41] A. Roberge, Phys. Rev. D 491689 (1994).

[42] V. A. Rubakov and M. E. Shaposhnikov, Usp. Fiz. Nauk 166, 493 (1996) [Phys. Usp. 39, 461 (1996)]; hep-ph/9603208.

[43] A. Kovner, A. Krasnitz, R. Potting, Phys. Rev. D 61025009 (2000).

[44] P. Arnold, D. Son, L. Yaffe, Phys. Rev. D55, 6264 (1997) Phys. Rev. D59, 105020 (1999).

[45] D. Bödeker, Phys. Lett. B426, 351 (1998); Nucl. Phys B559, 502 (1999).

[46] J. Ambjørn, K.N. Anagnostopoulos, A. Krasnitz, JHEP 0106069 (2001). 
[47] A. Krasnitz, Y. Nara and R. Venugopalan hep-ph/0108092.

[48] A. Krasnitz, Y. Nara and R. Venugopalan, in progress.

[49] I. G. Bearden et al. [BRAHMS Collaborations] nucl-ex/0108016; I. G. Bearden et al. [BRAHMS Collaboration] Phys. Rev. Lett. 87, 112305 (2001); B. B. Back et al. [PHOBOS Collaboration] Phys. Rev. Lett. 87, 102303 (2001)

[50] M. Baker, private communication. 